Ternational Electronic Journal of Algebra

Volume 29 (2021) 120-133

DOI: $10.24330 /$ ieja. 772801

\title{
SOME ALGEBRAS IN TERMS OF DIFFERENTIAL OPERATORS
}

\author{
Ghorban Soleymanpour and Ali S. Janfada \\ Received: 16 February 2020; Revised: 1 May 2020; Accepted 27 May 2020 \\ Communicated by Abdullah Harmancı \\ Abstract. Let $C$ be a commutative ring and $C\left[x_{1}, x_{2}, \ldots\right]$ the polynomial ring \\ in a countable number of variables $x_{i}$ of degree 1 . Suppose that the differential \\ operator $d^{1}=\sum_{i} x_{i} \partial_{i}$ acts on $C\left[x_{1}, x_{2}, \ldots\right]$. Let $\mathbb{Z}_{p}$ be the $p$-adic integers, $K$ \\ the extension field of the $p$-adic numbers $\mathbb{Q}_{p}$, and $\mathbb{F}_{2}$ the 2 -element filed. In this \\ article, first, the $C$-algebra $\mathcal{A}_{1}(C)$ of differential operators is constructed by \\ the divided differential operators $\left(d^{1}\right)^{\vee k} / k$ ! as its generators, where $\vee$ stands \\ for the wedge product. Then, the free Baxter algebra of weight 1 over $\varnothing$, \\ the $\lambda$-divided power Hopf algebra $\mathcal{A}_{\lambda}$, the algebra $C\left(\mathbb{Z}_{p}, K\right)$ of continuous \\ functions from $\mathbb{Z}_{p}$ to $K$, and the algebra of all $\mathbb{F}_{2}$-valued continuous functions \\ on the ternary Cantor set are represented in terms of the differential operators \\ algebra $\mathcal{A}_{1}(C)$.
}

Mathematics Subject Classification (2020): 13N10, 55S10

Keywords: Differential operator, integral Steenrod operator, $\lambda$-divided power Hopf algebra, Baxter algebra

\section{Introduction}

In [11], Wood considered the differential operators

$$
D_{k}=\sum_{i} x_{i}^{k+1} \frac{\partial}{\partial x_{i}}, k \geq 1,
$$

acting in the usual way on the integral polynomial ring $\mathbb{Z}\left[x_{1}, x_{2}, \ldots\right]$ in a countable number of variables $x_{i}$ of degree 1 in order to give an introductory presentation of the Steenrod algebra from a purely algebraic point of view.

The differential operators $D_{k}$ have been known to topologists for a long time. These operators form an algebra, under the wedge product $\vee$, generated by the divided differential operators $D_{k}^{\vee r} / r$ !. Wood named this algebra as the divided differential operator algebra $\mathcal{D}$. Moreover, the algebra $\mathcal{D}$ is closed under the composition of operators and is isomorphic to the Landweber-Novikov algebra due to this multiplication. Interpretations of the Landweber-Novikov algebra in terms of differential operators have been offered in the works of Buhstaber and the others $[3,4,5]$. 
In this article, it is assumed that $C$ is a commutative ring. Let $C\left[x_{1}, x_{2}, \ldots\right]$ denote the polynomial ring in a countable number of variables $x_{i}$ of degree 1 . Take the differential operator

$$
d^{1}=\sum_{i} x_{i} \partial_{i}
$$

acting on $C\left[x_{1}, x_{2}, \ldots\right]$. The operator $D_{0}$ is familiar from Euler's formula

$$
D_{0}(f)=\sum_{i} x_{i} \frac{\partial f}{\partial x_{i}}=(\operatorname{deg} f) f
$$

for any homogeneous polynomial $f$. Wood considered $D_{0}$ as the identity operator. However, considering $D_{0}$ as a non-identity operator, we define a specific differential operators algebra $\mathcal{A}_{1}(C)$ generated by the divided differential operators $\left(d^{1}\right)^{\vee k} / k$ ! which has certain properties. In particular, representations of several algebras are expressed in the literature of the differential operators algebra $\mathcal{A}_{1}(C)$. Given a prime $p$ we take $\mathbb{Z}_{p}$ the $p$-adic integers and $\mathbb{Q}_{p}$ the field of $p$-adic numbers.

(1) The free Baxter algebra of weight 1 over $\varnothing$ is isomorphic to the $C$-algebra $\mathcal{A}_{1}(C)$

(2) The $\lambda$-divided power Hopf algebra $\mathcal{A}_{\lambda}$ [1] is represented by a suitable multiple of generators of $\mathcal{A}_{1}(C)$;

(3) For a field $\mathbb{F}$ of characteristic 0 , the $\mathbb{F}$-algebra $\mathcal{A}_{1}(\mathbb{F})$ is a polynomial algebra. In particular, for the extension $K$ of $\mathbb{Q}_{p}$, the completion $\widehat{\mathcal{A}}_{1}(K)$ of $\mathcal{A}_{1}(K)$ with the max-norm is isomorphic to $\mathcal{C}\left(\mathbb{Z}_{p}, K\right)$, the algebra of continuous functions from $\mathbb{Z}_{p}$ to $K$;

(4) For the two-element field $\mathbb{F}_{2}$, the algebra $\mathcal{A}_{1}\left(\mathbb{F}_{2}\right)$ is isomorphic to the algebra of all the $\mathbb{F}_{2}$-valued continuous functions on the ternary Cantor set.

\section{The 1-Steenrod algebra}

Let $I=\left(i_{1}^{r_{1}}, i_{2}^{r_{2}}, \ldots, i_{n}^{r_{n}}\right)$ and $K=\left(k_{1}^{s_{1}}, k_{2}^{s_{2}}, \ldots, k_{n}^{s_{n}}\right)$ be the multisets of positive integers and put

$$
\partial_{i}^{k}=\frac{\partial^{k}}{\partial x_{i}^{k}}
$$

We write abbreviated expressions for monomials

$$
x_{I}=x_{i_{1}}^{r_{1}} x_{i_{2}}^{r_{2}} \cdots x_{i_{n}}^{r_{n}}, \partial_{K}=\partial_{k_{1}}^{s_{1}} \partial_{k_{2}}^{s_{2}} \cdots \partial_{k_{n}}^{s_{n}} .
$$

The degree of $x_{I}$ and the order of $\partial_{K}$ are $r_{1}+r_{2}+\cdots+r_{n}$ and $s_{1}+s_{2}+\cdots+s_{n}$, respectively. Based on [11], we adopt the wedge symbol $\vee$ for the formal product of two differential operators $x_{I} \partial_{K}$ and $x_{J} \partial_{L}$ defined on $C\left[x_{1}, x_{2}, \ldots\right]$ by

$$
x_{I} \partial_{K} \vee x_{J} \partial_{L}=x_{I} x_{J} \partial_{K} \partial_{L} .
$$


Our work draws upon the differential operators of the form $x_{I} \partial_{I}$, for the multiset $I=\left(i_{1}, \ldots, i_{n}\right)$. Therefore, we need only the special differential operator

$$
d^{1}=\sum_{i} x_{i} \partial_{i}
$$

acting on $C\left[x_{1}, x_{2}, \ldots\right]$. By the above notation,

$$
d^{1} \vee d^{1}=\sum_{i, j} x_{i} x_{j} \partial_{i j}
$$

and, generally, for the multiset $I=\left(i_{1}, i_{2}, \ldots, i_{k}\right)$,

$$
\left(d^{1}\right)^{\vee k}=\overbrace{d^{1} \vee \cdots \vee d^{1}}^{k \text { times }}=\sum_{I} x_{I} \partial_{I}=\sum_{\left(i_{1}, \ldots, i_{k}\right)} x_{i_{1}} \cdots x_{i_{k}} \frac{\partial^{k}}{\partial x_{i_{1}} \cdots \partial x_{i_{k}}} .
$$

Leibniz formula yields for the above operation.

Lemma 2.1 (Leibniz formula). For any polynomials $f, g \in C\left[x_{1}, x_{2}, \ldots\right]$,

$$
\left(d^{1}\right)^{\vee n}(f g)=\sum_{s=0}^{n}\left(\begin{array}{l}
n \\
s
\end{array}\right)\left(d^{1}\right)^{\vee s}(f)\left(d^{1}\right)^{\vee(n-s)}(g) .
$$

Proof. First, note that for $f, g \in C\left[x_{1}, x_{2}, \ldots\right]$, we have

$$
\frac{\partial^{n}(f g)}{\partial x_{i_{1}} \cdots \partial x_{i_{n}}}=\sum_{S} \frac{\partial^{|S|}(f)}{\prod_{i_{k} \in S} \partial x_{i_{k}}} \frac{\partial^{n-|S|}(g)}{\prod_{i_{k} \notin S} \partial x_{i_{k}}},
$$

where the summation runs over all subsets $S$ of the set $\left\{i_{1}, \ldots, i_{n}\right\}$. For $S=$ $\left\{i_{r_{1}}, \ldots, i_{r_{s}}\right\}$, denote by $(S)$ the multiset $\left(i_{r_{1}}, \ldots, i_{r_{s}}\right)$ of $s$-tuples of elements of $\left\{i_{1}, \ldots, i_{n}\right\}$. As before, put $I=\left(i_{1}, \ldots, i_{n}\right)$. Then, we can rewrite $(1)$ in terms of the multisets as

$$
\partial_{I}(f g)=\sum_{S} \partial_{(S)}(f) \partial_{\left(S^{\prime}\right)}(g)
$$

where $S^{\prime}$ is the complement of $S$ in $\left\{i_{1}, \ldots, i_{n}\right\}$. Accordingly,

$$
\begin{aligned}
\left(d^{1}\right)^{\vee n}(f g) & =\sum_{I} x_{I} \partial_{I}(f g) \\
& =\sum_{I} x_{I} \sum_{S} \partial_{(S)}(f) \partial_{\left(S^{\prime}\right)}(g) .
\end{aligned}
$$

For an arbitrary subset $S$ of $\left\{i_{1}, \ldots, i_{n}\right\}$, we have

$$
\begin{aligned}
\sum_{I} x_{I} \partial_{(S)}(f) \partial_{\left(S^{\prime}\right)}(g) & =\sum_{I}\left(x_{(S)} \partial_{(S)}(f)\right)\left(x_{\left(S^{\prime}\right)} \partial_{\left(S^{\prime}\right)}(g)\right) \\
& =\left(d^{1}\right)^{\vee|S|}(f)\left(d^{1}\right)^{\vee(n-|S|)}(g) .
\end{aligned}
$$

Since there are exactly $\left(\begin{array}{l}n \\ s\end{array}\right)$ subsets of $\left\{i_{1}, \ldots, i_{n}\right\}$ of size $|S|=s$, the result follows. 
By applying the Leibniz formula to $x f$, where $x$ is linear, and using induction on the degree of $f$, it follows that $\left(d^{1}\right)^{\vee k}$ is divisible by $k$ ! for $k>0$. Consequently, it makes sense to consider the differential operators

$$
d^{k}:=\frac{\left(d^{1}\right)^{\vee k}}{k !} \text { for } k>0, d^{0}:=\mathrm{id} .
$$

Proposition 2.2. The differential operators $d^{k}, k>0$, have the following properties.

(1) For any non-negative integer $r$ and single variable $x_{j}$,

$$
d^{k}\left(x_{j}^{r}\right)= \begin{cases}\left(\begin{array}{l}
r \\
k
\end{array}\right) x_{j}^{r}, & \text { if } k \leq r \\
0, & \text { if } k>r\end{cases}
$$

(2) (Cartan formula) For any polynomials $f, g \in C\left[x_{1}, x_{2}, \ldots\right]$,

$$
d^{k}(f g)=\sum_{i+j=k} d^{i}(f) d^{j}(g)
$$

Proof. By definition, for $x_{j}^{r}$ we have

$$
d^{k}\left(x_{j}^{r}\right)=\frac{1}{k !} \sum_{\left(i_{1}, \ldots, i_{k}\right)} x_{i_{1}} \cdots x_{i_{k}} \frac{\partial^{k}}{\partial x_{i_{1}} \cdots \partial x_{i_{k}}}\left(x_{j}^{r}\right),
$$

which is 0 except for the index $(j, j, \ldots, j)$. Thus,

$$
d^{k}\left(x_{j}^{r}\right)=\frac{1}{k !} x_{j}^{k} \frac{\partial^{k}}{\left(\partial x_{j}\right)^{k}}\left(x_{j}^{r}\right)= \begin{cases}\left(\begin{array}{l}
r \\
k
\end{array}\right) x_{j}^{r}, & \text { if } k \leq r, \\
0, & \text { if } k>r .\end{cases}
$$

To deduce the Cartan formula, for $f, g \in C\left[x_{1}, x_{2}, \ldots\right]$ we write

$$
\frac{\left(d^{1}\right)^{\vee k}}{k !}=\sum_{i+j=k} \frac{\left(d^{1}\right)^{\vee i}}{i !}(f) \frac{\left(d^{1}\right)^{\vee j}}{j !}(g),
$$

using Lemma 2.1. This completes the proof.

Definition 2.3. The $C$-algebra generated by the set $\left\{d^{k}\right\}_{k \geq 0}$, under the composition of the differential operators $d^{k}$, is called the 1-Steenrod algebra over $C$ and denoted by $\mathcal{A}_{1}(C)$.

For example, $d^{1} d^{1}$ is computed in $\mathcal{A}_{1}(C)$ as follow.

$$
d^{1} d^{1}=\sum_{i} x_{i} \partial_{i}\left(\sum_{j} x_{j} \partial_{j}\right)=\sum_{i, j} x_{i} \partial_{i}\left(x_{j}\right) \partial_{j}+\sum_{i, j} x_{i} x_{j} \partial_{i j}=d^{1}+2 d^{2} .
$$


Remark 2.4. The mod 2 Steenrod algebra $\mathcal{A}_{2}$ is representable in terms of the differential operators

$$
S Q^{k}=\frac{D_{1}^{\vee k}}{k !}
$$

where $D_{1}=\sum_{i} x_{i}^{2} \partial_{i}$ [11]. However, instead of the squares of the variables $x_{i}$, in the present article, we deal with their first powers. This is the reason for selecting the notation $\mathcal{A}_{1}(C)$ and calling it the 1 -Steenrod algebra.

Given the positive integers $m$ and $n$, the general product $d^{m} d^{n}$ in $\mathcal{A}_{1}(C)$ is determined in the next result. We use the multiset notations in the proof of Lemma 2.1 .

Theorem 2.5. In $\mathcal{A}_{1}(C)$, we have

$$
d^{m} d^{n}=\sum_{s=0}^{m}\left(\begin{array}{c}
m \\
s
\end{array}\right)\left(\begin{array}{c}
m+n-s \\
m
\end{array}\right) d^{m+n-s} .
$$

In particular, $\mathcal{A}_{1}(C)$ is commutative.

Proof. All we need is to calculate the value

$$
d^{m} d^{n}=\frac{1}{m ! n !} \sum_{I} x_{I} \partial_{I}\left(\sum_{J} x_{J} \partial_{J}\right)=\frac{1}{m ! n !} \sum_{I, J} x_{I} \partial_{I}\left(x_{J} \partial_{J}\right) .
$$

We know that

$$
\partial_{I}\left(x_{J} \partial_{J}\right)=\sum_{S} \partial_{(S)}\left(x_{J}\right) \partial_{\left(S^{\prime}\right)}\left(\partial_{J}\right)
$$

Let $m \leq n$. Then,

$$
d^{m} d^{n}=\frac{1}{m ! n !} \sum_{I, J} x_{I} \sum_{S} \partial_{(S)}\left(x_{J}\right) \partial_{\left(S^{\prime}\right)}\left(\partial_{J}\right)
$$

For any individual subset $S$, we compute the $S$-summation. In the case $|S|=0$, we have the $S$-summand

$$
\frac{1}{m ! n !} \sum_{I, J} x_{I} x_{J} \partial_{I} \partial_{J}=\frac{(m+n) !}{m ! n !} d^{m+n}=\left(\begin{array}{c}
m \\
0
\end{array}\right)\left(\begin{array}{c}
m+n-0 \\
m
\end{array}\right) d^{m+n} .
$$

If $|S|=s>0$, then $S=\left\{i_{r_{1}}, \ldots, i_{r_{s}}\right\}$ and the $S$-summand is

$$
\frac{1}{m ! n !} \sum_{I, J} x_{I} \partial_{(S)}\left(x_{J}\right) \partial_{\left(S^{\prime}\right)} \partial_{J}
$$

where $\partial_{(S)}\left(x_{J}\right)$ is nonzero only if $i_{r_{1}}, \ldots, i_{r_{s}}$ are components of $J$ in which case,

$$
\partial_{(S)}\left(x_{J}\right)=\frac{x_{J}}{x_{(S)}}
$$


However, there are $n(n-1) \cdots(n-s+1)$ possibilities for $i_{r_{1}}, \ldots, i_{r_{s}}$ to be components of $J$. Therefore, the $S$-summand (3) turns to

$$
\begin{aligned}
\frac{n(n-1) \cdots(n-s+1)}{m ! n !} \sum_{I, J} x_{I} \frac{x_{J}}{x_{(S)}} \partial_{\left(S^{\prime}\right)} \partial_{J} & \\
& =n(n-1) \cdots(n-s+1) \frac{(m+n-s) !}{m ! n !} d^{m+n-s} \\
& =\left(\begin{array}{c}
m+n-s \\
m
\end{array}\right) d^{m+n-s} .
\end{aligned}
$$

On the other hand, there are $\left(\begin{array}{c}m \\ s\end{array}\right)$ subsets of size $|S|=s$. We can conclude that the coefficient of $d^{m+n-s}$ in the right hand of (2) is

$$
\left(\begin{array}{c}
m \\
s
\end{array}\right)\left(\begin{array}{c}
m+n-s \\
m
\end{array}\right)
$$

Now, for $0 \leq s \leq m$,

$$
\left(\begin{array}{c}
m \\
s
\end{array}\right)\left(\begin{array}{c}
m+n-s \\
m
\end{array}\right)=\left(\begin{array}{c}
n \\
s
\end{array}\right)\left(\begin{array}{c}
m+n-s \\
n
\end{array}\right)
$$

while for $m<s \leq n$ the coefficient $\left(\begin{array}{c}m+n-s \\ n\end{array}\right)$ annihilates. Therefore, $\mathcal{A}_{1}(C)$ is commutative.

\section{Baxter algebra and the Hopf algebra structure of $\mathcal{A}_{1}(C)$}

Definition 3.1. A commutative $C$-algebra $B$ is called a Baxter algebra of weight $\lambda \in C$, if there exists a $C$-linear operator $T: B \rightarrow B$ such that for all $x, y \in B$,

$$
T(x) T(y)=T(x T(y))+T(y T(x))+\lambda T(x y) .
$$

The operator $T$ is called a Baxter operator of weight $\lambda$.

Theorem 3.2. The map $T: \mathcal{A}_{1}(C) \rightarrow \mathcal{A}_{1}(C)$ defined by $T\left(d^{n}\right)=d^{n+1}$ on the generators $d^{n}$ is a Baxter operator of weight 1 .

Proof. For the sake of simplicity, denote the coefficient $\left(\begin{array}{c}m \\ s\end{array}\right)\left(\begin{array}{c}m+n-s \\ m\end{array}\right)$ by $R_{m}^{n}(s)$. For $m, n \geq 1$, calculate

$$
\begin{aligned}
A & =T\left(d^{m} T\left(d^{n}\right)\right)+T\left(d^{n} T\left(d^{m}\right)\right)+T\left(d^{m} d^{n}\right) \\
& =T\left(d^{m} d^{n+1}\right)+T\left(d^{m+1} d^{n}\right)+T\left(d^{m} d^{n}\right)
\end{aligned}
$$


as follows.

$$
\begin{aligned}
A= & \sum_{s=0}^{m} R_{m}^{n+1}(s) d^{m+n+2-s}+\sum_{s=0}^{m+1} R_{m+1}^{n}(s) d^{m+n+2-s}+\sum_{s=0}^{m} R_{m}^{n}(s) d^{m+n+1-s} \\
= & {\left[\left(\begin{array}{c}
m+n+1 \\
m
\end{array}\right)+\left(\begin{array}{c}
m+n+1 \\
m+1
\end{array}\right)\right] d^{m+n+2}+\left[\left(\begin{array}{c}
n \\
m+1
\end{array}\right)+\left(\begin{array}{c}
n \\
m
\end{array}\right)\right] d^{n+1} } \\
& +\sum_{s=1}^{m}\left(R_{m}^{n+1}(s)+R_{m+1}^{n}(s)+R_{m}^{n}(s-1)\right) d^{m+n+2-s} \\
= & \left(\begin{array}{c}
m+n+2 \\
m+1
\end{array}\right) d^{m+n+2}+\left(\begin{array}{c}
n+1 \\
m+1
\end{array}\right) d^{n+1} \\
& +\sum_{s=1}^{m}\left(\begin{array}{c}
m+1 \\
s
\end{array}\right)\left(\begin{array}{c}
m+n+2-s \\
m
\end{array}\right) d^{m+n+2-s} \\
= & d^{m+1} d^{n+1} \\
= & T\left(d^{m}\right) T\left(d^{n}\right),
\end{aligned}
$$

showing that $T$ is a Baxter operator of weight 1 .

Additionally, $\mathcal{A}_{1}(C)$ is a free Baxter algebra of weight 1 over $X=\varnothing[6]$. Generally, for $\lambda \in C$, let

$$
\mathcal{A}_{\lambda}(C)=\bigoplus_{n=0}^{\infty} C a_{n}
$$

be the free $C$-module over the set $\left\{a_{n}\right\}_{n \geq 0}$. Then the map

$$
\mu_{\lambda}: \mathcal{A}_{\lambda}(C) \otimes_{C} \mathcal{A}_{\lambda}(C) \rightarrow \mathcal{A}_{\lambda}(C),
$$

defined by

$$
\mu_{\lambda}\left(a_{m} \otimes a_{n}\right)=\sum_{s=0}^{m} \lambda^{s}\left(\begin{array}{c}
m \\
s
\end{array}\right)\left(\begin{array}{c}
m+n-s \\
m
\end{array}\right) a_{m+n-s},
$$

provides a multiplication on $\mathcal{A}_{\lambda}(C)$ subject to $a_{0}=1$. Now, the operator

$$
\begin{aligned}
T: \mathcal{A}_{\lambda}(C) & \rightarrow \mathcal{A}_{\lambda}(C), \\
a_{n} & \mapsto a_{n+1}
\end{aligned}
$$

is a Baxter operator of weight $\lambda$. The Hopf algebra structure of $\mathcal{A}_{\lambda}(C)$ is given by the next result [1, Theorem 1.1].

Theorem 3.3. The algebra $\mathcal{A}_{\lambda}(C)$ is a Hopf algebra with the diagonal map $\Delta$ : $\mathcal{A}_{\lambda}(C) \rightarrow \mathcal{A}_{\lambda}(C) \otimes_{C} \mathcal{A}_{\lambda}(C)$ defined by

$$
\Delta\left(a_{n}\right)=\sum_{k=0}^{n} \sum_{j=0}^{n-k}(-\lambda)^{k} a_{j} \otimes a_{n-k-j},
$$


and the counit $\varepsilon: \mathcal{A}_{\lambda}(C) \rightarrow \mathcal{A}_{\lambda}(C)$ defined by

$$
\varepsilon\left(a_{n}\right)= \begin{cases}1, & \text { if } n=0, \\ \lambda 1, & \text { if } n=1, \\ 0, & \text { if } n \geq 2\end{cases}
$$

Whenever $\lambda=0$, we have the divided power Hopf algebra $\mathcal{A}_{0}(C)$ in which, $a_{m} a_{n}=\left(\begin{array}{c}m+n \\ m\end{array}\right) a_{m+n}$. In general, $\mathcal{A}_{\lambda}(C)$ is called the $\lambda$-divided power Hopf algebra. The divided power algebra $\mathcal{A}_{0}(C)$ plays an important role in several areas of mathematics, including the crystalline cohomology in number theory [2], the umbral calculus in combinatorics [8], and the Hurwitz series in differential algebra [7].

In the next theorem, we provide a representation of the algebra $\mathcal{A}_{\lambda}(C)$ as an algebra of differential operators.

Theorem 3.4. Consider the operators

$$
\begin{aligned}
d_{\lambda}^{n} & =\frac{\lambda^{n}}{n !} \sum_{\left(i_{1}, \ldots, i_{n}\right)} x_{i_{1}} \cdots x_{i_{n}} \frac{\partial^{n}}{\partial x_{i_{1}} \cdots \partial x_{i_{n}}}=\lambda^{n} d^{n}, \quad \lambda \neq 0, \\
d_{0}^{n} & =\frac{1}{n !} \sum_{\left(i_{1}, \ldots, i_{n}\right)} \frac{\partial^{n}}{\partial x_{i_{1}} \cdots \partial x_{i_{n}}} .
\end{aligned}
$$

Then, the $C$-algebra generated by the set $\left\{d_{\lambda}^{n}\right\}_{n \geq 0}$, under composition, is isomorphic to $\mathcal{A}_{\lambda}(C)$.

Proof. We examine the multiplication of the elements $d_{\lambda}^{m}$ and $d_{\lambda}^{n}$. For $\lambda \neq 0$, we have

$$
\begin{aligned}
d_{\lambda}^{m} d_{\lambda}^{n} & =\lambda^{m+n} d^{m} d^{n} \\
& =\sum_{s=0}^{m} \lambda^{m+n}\left(\begin{array}{c}
m \\
s
\end{array}\right)\left(\begin{array}{c}
m+n-s \\
m
\end{array}\right) d^{m+n-s} \\
& =\sum_{s=0}^{m}\left(\begin{array}{c}
m \\
s
\end{array}\right)\left(\begin{array}{c}
m+n-s \\
m
\end{array}\right) \lambda^{s} d_{\lambda}^{m+n-s} \\
& =d_{\lambda}^{m} d_{\lambda}^{n} .
\end{aligned}
$$


And for $\lambda=0$,

$$
\begin{aligned}
d_{0}^{m} d_{0}^{n} & =\frac{1}{m ! n !} \sum_{\substack{\left(i_{1}, \ldots, i_{m}\right)\\
}} \frac{\partial^{m}}{\partial x_{i_{1}} \cdots \partial x_{i_{m}}} \sum_{\left(j_{1}, \ldots, j_{n}\right)} \frac{\partial^{n}}{\partial x_{j_{1}} \cdots \partial x_{j_{n}}} \\
& =\frac{1}{m ! n !} \sum_{\substack{\left(i_{1}, \ldots, i_{m}\right) \\
\left(j_{1}, \ldots, j_{n}\right)}} \frac{\partial^{m+n}}{\partial x_{i_{1}} \cdots \partial x_{i_{n}} \partial x_{j_{1}} \cdots \partial x_{j_{m}}} \\
& =\left(\begin{array}{c}
m+n \\
m
\end{array}\right) d_{0}^{m+n} .
\end{aligned}
$$

This establishes the theorem.

Therefore, the functor $\mathcal{A}_{\lambda}(-)$, associates any commutative ring $C$ with the Baxter algebra $\mathcal{A}_{\lambda}(C)$ of weight $\lambda$ and any ring homomorphism $\phi: C \rightarrow C^{\prime}$ with the algebra homomorphism

$$
\begin{aligned}
\mathcal{A}_{\lambda}(\phi): \mathcal{A}_{\lambda}(C) & \rightarrow \mathcal{A}_{\lambda}\left(C^{\prime}\right), \\
\sum_{i=1}^{n} a_{i} d^{k_{i}} & \mapsto \sum_{i=1}^{n} \phi\left(a_{i}\right) d^{k_{i}} .
\end{aligned}
$$

\section{Representation of $\mathcal{C}\left(\mathbb{Z}_{p}, K\right)$ in terms of differential operators}

Over a field $\mathbb{F}$ of characteristic 0 , the algebra $\mathcal{A}_{1}(\mathbb{F})$ is familiar.

Theorem 4.1. Let $\mathbb{F}$ be a field of characteristic 0 . Then $\mathcal{A}_{1}(\mathbb{F})$ is isomorphic to the polynomial algebra $\mathbb{F}[t]$.

Proof. By Theorem 2.5, we have $d^{1} d^{1}=d^{1}+2 d^{2}$ or $d^{2}=\frac{1}{2} d^{1}\left(d^{1}-1\right)$. By induction on $n$ we get

$$
d^{n}=\frac{1}{k !} d^{1}\left(d^{1}-1\right) \cdots\left(d^{1}-n+1\right)
$$

Now consider the morphism $\mathcal{A}_{1}(\mathbb{F}) \rightarrow \mathbb{F}[t]$, defined by

$$
d^{n} \mapsto \frac{t(t-1) \cdots(t-n+1)}{n !} .
$$

This map is an isomorphism of $\mathbb{F}$-algebras since the $\mathbb{F}[t]$ is generated by the polynomials $\frac{t(t-1) \cdots(t-n+1)}{n !}$ for $n \geq 1$. In fact, we have

$$
t^{n}=\sum_{k=0}^{n} a_{k} \frac{t(t-1) \cdots(t-k+1)}{k !}
$$

where

$$
a_{k}=\sum_{i=1}^{k}(-1)^{k-i}\left(\begin{array}{c}
k \\
i
\end{array}\right) i^{k}
$$

for all $0 \leq k \leq n[9$, Section 52]. This completes the proof. 
Using Theorems 2.5 and 4.1, one can see that

$$
\left(\begin{array}{l}
x \\
m
\end{array}\right)\left(\begin{array}{l}
x \\
n
\end{array}\right)=\sum_{s=0}^{m}\left(\begin{array}{c}
m \\
s
\end{array}\right)\left(\begin{array}{c}
m+n-s \\
m
\end{array}\right)\left(\begin{array}{c}
x \\
m+n-s
\end{array}\right) .
$$

In particular,

$$
\left(\begin{array}{c}
x \\
m
\end{array}\right)^{2}=\sum_{s=0}^{m}\left(\begin{array}{c}
m \\
s
\end{array}\right)\left(\begin{array}{c}
2 m-s \\
m
\end{array}\right)\left(\begin{array}{c}
x \\
2 m-s
\end{array}\right) .
$$

Definition 4.2. For $n \geq 0$, the polynomial $F_{n}(x)$ is defined by

$$
F_{n}(x)=\left(\begin{array}{l}
x \\
n
\end{array}\right)=\frac{x(x-1) \cdots(x-n+1)}{n !} \text { for } n>0, \text { and } F_{0}(x)=1 .
$$

Suppose that $\mathcal{C}\left(\mathbb{Z}_{p}, K\right)$ is the set of all continuous maps from the $p$-adic integers $\mathbb{Z}_{p}$ to the extension $K$ of the field of $p$-adic numbers $\mathbb{Q}_{p}$. Under the point-wise addition and multiplication

$$
\begin{gathered}
(f+g)(x)=f(x)+g(x), \\
(f g)(x)=f(x) g(x),
\end{gathered}
$$

for all $f, g \in \mathcal{C}\left(\mathbb{Z}_{p}, K\right)$ and $x \in \mathbb{Z}_{p}$, the set $\mathcal{C}\left(\mathbb{Z}_{p}, K\right)$ forms a $K$-algebra. Furthermore, $\mathcal{C}\left(\mathbb{Z}_{p}, K\right)$ is a Banach algebra subject to the sup-norm

$$
\|f\|_{\infty}:=\sup _{x \in \mathbb{Z}_{p}}|f(x)|
$$

where || denotes the absolute value of the field $K$ (see [9, Section 13]). The next result is a combination of Theorems 51.1 and 52.1 of [9].

Theorem 4.3. Let $K$ be an extension of the field of $p$-adic numbers with absolute value $\mid$.

(1) (Mahler expansion). For $f \in \mathcal{C}\left(\mathbb{Z}_{p}, K\right)$, there exist unique elements $a_{0}, a_{1}, \ldots$ of $K$, called Mahler coefficients of $f$, such that

$$
f(x)=\sum_{n=0}^{\infty} a_{n} F_{n}(x) .
$$

This series is called the Mahler expansion of $f$ and converges uniformly. Moreover, the sup-norm of $f$ is calculated by

$$
\|f\|_{\infty}=\sup _{n \geq 0}\left|a_{n}\right| .
$$

(2) If $a_{0}, a_{1}, \ldots$ is a null sequence in $K$, i.e., if $\lim _{n \rightarrow \infty}\left|a_{n}\right|=0$, then $x \mapsto$ $\sum_{n=0}^{\infty} a_{n} F_{n}(x)$ defines a continuous function $\mathbb{Z}_{p} \rightarrow K$. 
(3) For $f \in \mathcal{C}\left(\mathbb{Z}_{p}, K\right)$ with Mahler expansion $f=\sum_{n=0}^{\infty} a_{n} F_{n}$, the coefficients $a_{n}$ can be reconstructed from $f$ by

$$
a_{n}=\sum_{j=0}^{n}(-1)^{n-j}\left(\begin{array}{l}
n \\
j
\end{array}\right) f(j) .
$$

For example, using the equation (4), we can see that, for a fixed $m$,

$$
\sum_{j=0}^{n}(-1)^{n-j}\left(\begin{array}{c}
n \\
j
\end{array}\right)\left(\begin{array}{c}
j \\
m
\end{array}\right)^{2}=0,
$$

for all $n<m$ and $n>2 m$.

By Theorem 4.3, we have $\left\|F_{n}\right\|=1$ for $n \geq 0$. Using the isomorphism $d^{n} \mapsto F_{n}$, we can define a norm on $\mathcal{A}_{1}(K)$ by $\left\|d^{n}\right\|=1$ for all $n \geq 0$ and for an arbitrary element $\theta=\sum_{i=1}^{r} a_{i} d^{n_{i}} \in \mathcal{A}_{1}(K)$,

$$
\|\theta\|=\sup _{1 \leq i \leq r}\left|a_{i}\right| .
$$

Denote by $\widehat{\mathcal{A}}_{1}(K)$ the completion of the normed algebra $\mathcal{A}_{1}(K)$ consisting of all infinite series $\sum_{n=0}^{\infty} a_{n} d^{n}$ with $\lim _{n \rightarrow \infty}\left|a_{n}\right|=0$. Then, the following corollary is clear.

Corollary 4.4. $\mathcal{C}\left(\mathbb{Z}_{p}, K\right)$ is isomorphic to $\widehat{\mathcal{A}}_{1}(K)$.

\section{5. $\{0,1\}$-valued continuous functions on the ternary Cantor set}

In this section, taking $P$ as the classic ternary Cantor set, we present the $\mathbb{F}_{2}-$ algebra $\mathcal{C}\left(P, \mathbb{F}_{2}\right)$ in terms of differential operators. To this end, first we concentrate on $\mathcal{A}_{1}\left(\mathbb{F}_{2}\right)$.

Theorem 5.1. Every element of $\mathcal{A}_{1}\left(\mathbb{F}_{2}\right)$ is idempotent. Additively, the collection $\left\{d^{2^{n}}\right\}_{n \geq 0}$ generates the $\mathbb{F}_{2}$-algebra $\mathcal{A}_{1}\left(\mathbb{F}_{2}\right)$ and for the positive integer $n$ with binary expansion $n=2^{j_{1}}+\cdots+2^{j_{r}}$,

$$
d^{n}=d^{2^{j_{1}}} \cdots d^{2^{j_{r}}}
$$

To prove the theorem, we need the following lemma [10, Proposition 1.4.11]. For the positive integer $d=2^{d_{1}}+\cdots+2^{d_{r}}$, define $\operatorname{bin}(\mathrm{d})=\left\{2^{\mathrm{d}_{1}}, \ldots, 2^{\mathrm{d}_{\mathrm{r}}}\right\}$ and put $\operatorname{bin}(0)=\varnothing$.

Lemma 5.2. For $a, b \geq 0,\left(\begin{array}{l}b \\ a\end{array}\right) \equiv 1(\bmod 2)$ if and only if $\operatorname{bin}(\mathrm{a}) \subseteq \operatorname{bin}(\mathrm{b})$. 
Proof of Theorem 5.1. For $n \geq 1$, by Theorem 2.5, we calculate $d^{n} d^{n}$ in $\mathcal{A}_{1}\left(\mathbb{F}_{2}\right)$ as follows.

$$
d^{n} d^{n}=\sum_{s=0}^{n}\left(\begin{array}{c}
n \\
s
\end{array}\right)\left(\begin{array}{c}
2 n-s \\
n
\end{array}\right) d^{2 n-s}=d^{n}+\sum_{s=0}^{n-1}\left(\begin{array}{l}
n \\
s
\end{array}\right)\left(\begin{array}{c}
2 n-s \\
n
\end{array}\right) d^{2 n-s} .
$$

On the other hand, for $0 \leq s<n$, we obtain

$$
\begin{aligned}
\left(\begin{array}{c}
n \\
s
\end{array}\right)\left(\begin{array}{c}
2 n-s \\
n
\end{array}\right) & =\frac{n !}{s !(n-s) !} \frac{(2 n-s) !}{n !(n-s) !} \\
& =\frac{(2(n-s)) !}{(n-s) !(n-s) !} \frac{(2 n-s) !}{s !(2(n-s)) !} \\
& =\left(\begin{array}{c}
2(n-s) \\
n-s
\end{array}\right)\left(\begin{array}{c}
2 n-s \\
s
\end{array}\right) \\
& =2\left(\begin{array}{c}
2(n-s)-1 \\
n-s-1
\end{array}\right)\left(\begin{array}{c}
2 n-s \\
s
\end{array}\right) \\
& \equiv 0 \quad(\bmod 2) .
\end{aligned}
$$

Thus, $d^{n} d^{n}=d^{n}$ in $\mathcal{A}_{1}\left(\mathbb{F}_{2}\right)$. Consider the product

$$
d^{2^{a}} d^{n}=\sum_{s=0}^{2^{a}}\left(\begin{array}{c}
2^{a} \\
s
\end{array}\right)\left(\begin{array}{c}
2^{a}+n-s \\
2^{a}
\end{array}\right) d^{2^{a}+n-s},
$$

where $2^{a} \notin \operatorname{bin}(\mathrm{n})$. By Lemma $5.2,\left(\begin{array}{c}2^{a} \\ s\end{array}\right) \equiv 1(\bmod 2)$, if and only if $\operatorname{bin}(\mathrm{s}) \subseteq$ $\operatorname{bin}\left(2^{\mathrm{a}}\right)$, if and only if $s=0$ or $2^{a}$. Therefor,

$$
d^{2^{a}} d^{n}=d^{2^{a}+n}+\left(\begin{array}{c}
n \\
2^{a}
\end{array}\right) d^{n}=d^{2^{a}+n}
$$

since $2^{a} \notin \operatorname{bin}(\mathrm{n})$. Let $k=2^{a_{1}}+\cdots+2^{a_{r}}$ be the binary expansion of the number $k$. Then, by the inductive use of (5) we obtain

$$
d^{k}=d^{2^{a_{1}}+\cdots+2^{a_{r}}}=d^{2^{a_{1}}} d^{2^{a_{2}}+\cdots+2^{a_{r}}}=\cdots=d^{2^{a_{1}}} \cdots d^{2^{a_{r}}} .
$$

To complete the proof, it suffices to show that the elements $d^{2^{n}}$, for $n \geq 0$, are indecomposable. Contrarily, suppose $d^{2^{n}}=d^{a} d^{b}$, for some $0<a, b<2^{n}$. Therefore, there exists an index $0 \leq s_{0} \leq a$ for which, $a+b-s_{0}=2^{n}$. By Theorem 2.5 , the coefficient of $d^{a+b-s_{0}}=d^{2^{n}}$ in $d^{a} d^{b}$ is $\left(\begin{array}{c}a \\ s_{0}\end{array}\right)\left(\begin{array}{c}2^{n} \\ a\end{array}\right)$ which is $1(\bmod 2)$ if and only if $\operatorname{bin}(\mathrm{a}) \subseteq\left\{2^{\mathrm{n}}\right\}$, or equivalently $a=0$ or $2^{n}$, contradicts the choice of $a$.

The ternary Cantor set $P$ consisting of all the real numbers in $[0,1]$ with the ternary expansion $\sum_{n=1}^{\infty} a_{n} 3^{-n}$, where $a_{n}=0$ or 2 for all $n$, is homeomorphic to the infinite product $\prod_{n=0}^{\infty} \mathbb{F}_{2}$. 
Suppose that $\mathcal{C}\left(P, \mathbb{F}_{2}\right)$ is the set of all continuous functions over $P$ with values in $\mathbb{F}_{2}=\{0,1\}$. The point-wise addition and multiplication

$$
\begin{gathered}
(f+g)(x)=f(x)+g(x), \\
(f g)(x)=f(x) g(x),
\end{gathered}
$$

for all $f, g \in \mathcal{C}\left(P, \mathbb{F}_{2}\right)$ and $x \in P$, equip the set $\mathcal{C}\left(P, \mathbb{F}_{2}\right)$ with a commutative $\mathbb{F}_{2}$-algebra structure. The next result confirms $\mathcal{A}_{1}\left(\mathbb{F}_{2}\right)$ as a representation of this algebra.

Theorem 5.3. $\mathcal{A}_{1}\left(\mathbb{F}_{2}\right) \cong \mathcal{C}\left(P, \mathbb{F}_{2}\right)$.

Proof. We investigate the elements of $\mathcal{C}\left(P, \mathbb{F}_{2}\right)$. For any $f \in \mathcal{C}\left(P, \mathbb{F}_{2}\right)$, define the subset

$$
A_{f}=\left\{\left(x_{i}\right)_{i \geq 0} \in P: f\left(\left(x_{i}\right)\right)=1\right\} .
$$

Since $f$ is continuous, $A_{f}$ is a clopen subset of $P$. Furthermore, $A_{f}$ completely determines the function $f$. In fact, the functions $f, g \in \mathcal{C}\left(P, \mathbb{F}_{2}\right)$ are equal if and only if $A_{f}=A_{g}$. Therefore, there is a one to one correspondence between the elements of $\mathcal{C}\left(P, \mathbb{F}_{2}\right)$ and the clopen subsets of $P$. It is noteworthy that

$$
A_{f g}=A_{f} \cap A_{g}, A_{f+g}=A_{f} \cup A_{g}-A_{f g}, A_{1-f}=A_{f}^{\prime}=P-A,
$$

where 1 is the function corresponds to the entire clopen set $P$ and $A_{f}^{\prime}$ stands for the complement of $A_{f}$ in $P$.

Let $A_{j}=\prod_{i=0}^{\infty} X_{i}$, where $X_{j}=\{1\}$ and for $i \neq j, X_{i}=\mathbb{F}_{2}$. Then, $A_{j}$ is clopen and any clopen subset of $P$ is a finite intersection of the $A_{j}$ 's. Consider the projections $\pi_{j}: P \rightarrow \mathbb{F}_{2}$, defined by $\pi_{j}\left(\left(x_{i}\right)\right)=x_{j}$, the $j$ th component of $\left(x_{i}\right)_{i \geq 0} \in P$. Then,

$$
A_{\pi_{j}}=\left\{\left(x_{i}\right)_{i \geq 0} \in P: f\left(\left(x_{i}\right)\right)=1\right\}=A_{j}, A_{1-\pi_{j}}=P-A_{j}=A_{j}^{\prime} .
$$

Therefore, any clopen subset of $P$ corresponds to a finite sum of the finite products of projection maps. Now, the map

$$
\pi_{n} \mapsto d^{2^{n}}, n \geq 0
$$

is clearly an isomorphism of algebras $\mathcal{C}\left(P, \mathbb{F}_{2}\right) \rightarrow \mathcal{A}_{1}\left(\mathbb{F}_{2}\right)$.

Acknowledgement: The first author had a research visit of Steklov Mathematical Institute at St. Petersburg, Russia. He would like to thank professor S. S. Podkorytov for his great help in developing Theorem 5.3 during this visit. 


\section{References}

[1] G. E. Andrews, L. Guo, W. Keigher and K. Ono, Baxter algebras and Hopf algebras, Trans. Amer. Math. Soc., 355(11) (2003), 4639-4656.

[2] P. Berthelot and A. Ogus, Notes on Crystalline Cohomology, Princeton University Press, 1978.

[3] V. M. Buchstaber, Semigroups of maps into groups, operator doubles, and complex cobordisms, Amer. Math. Soc. Transl. Ser. 2, 170 (1995), 9-35.

[4] V. M. Buchstaber and A. N. Kholodov, Groups of formal diffeomorphisms of the superline, generating functions for sequences of polynomials, and functional equations, Izv. Akad. Nauk SSSR Ser. Mat., 53(5) (1989), 944-970.

[5] V. M. Buchstaber and A. V. Shokurov, The Landweber-Novikov algebra and formal vector fields on the line, Funktsional. Anal. i Prilozhen., 12(3) (1978), $1-11$.

[6] L. Guo, Baxter algebras and the umbral calculus, Adv. in Appl. Math., 27 (2001), 405-426.

[7] W. Keigher, On the ring of Hurwitz series, Comm. Algebra, 25(6) (1997), 1845-1859.

[8] S. M. Roman and G. C. Rota, The umbral calculus, Adv. Math., 27(2) (1978), 95-188.

[9] W. H. Schikhof, Ultrametric Calculus, Cambridge University Press, Cambridge, 1984.

[10] G. Walker and R. M. W. Wood, Polynomials and the mod-2 Steenrod Algebra, Volume 1, The Peterson hit problem, Cambridge University Press, Cambridge, 2018.

[11] R. M. W. Wood, Differential operators and the Steenrod algebra, Proc. Lond. Math. Soc. (3), 75(1) (1997), 194-220.

Gh. Soleymanpour and A. S. Janfada (Corresponding Author)

Department of Mathematics

Faculty of Science

Urmia University

Urmia 57561-51818, Iran

e-mails: gh.soleymanpour@urmia.ac.ir (Gh. Soleymanpour)

asjanfada@gmail.com; a.sjanfada@urmia.ac.ir (A. S. Janfada) 